\title{
9 Exograms and Interdisciplinarity: History, the Extended Mind, and the Civilizing Process
}

John Sutton

\section{Exograms, Interdisciplinarity, and the Cognitive Life of Things}

\subsection{The Extended Mind Hypothesis}

On the extended mind hypothesis (EM), ${ }^{1}$ many of our cognitive states and processes are hybrids, unevenly distributed across biological and nonbiological realms (Clark 1997; Clark and Chalmers 1998). In certain circumstances, things-artifacts, media, or technologies-can have a cognitive life, with histories often as idiosyncratic as those of the embodied brains with which they couple (Sutton 2002a, 2008). The realm of the mental can spread across the physical, social, and cultural environments as well as bodies and brains. My independent aims in this chapter are: first, to describe two compatible but distinct movements or "waves" within the EM literature, arguing for the priority of the second wave (and gesturing briefly toward a third); and, second, to defend and illustrate the interdisciplinary implications of EM as best understood, specifically for historical disciplines, by sketching two case studies.

EM, an offshoot of mainstream functionalist information-processing cognitive science, has been focused in particular on our abilities to hook up with what Merlin Donald calls "exograms" or external symbols, by analogy with the brain's memory traces or "engrams" (Donald 1991, pp. 308-333; 2001, pp. 305-315). ${ }^{2}$ These abilities allow us to create and support cognitive profiles quite unlike those of creatures restricted to the brain's biological memories or engrams alone. Among other typical features, Donald points out that exograms last longer than engrams, have greater capacity, are more easily transmissible across media and context, and can be retrieved and manipulated by a greater variety of means (1991, pp. 315-316): so our skilled use of such crafted aids changes both the locus of memory in general and the role of our biological 
memory within the new larger systems (see also Rowlands 1999, pp. 129-147).

As I'll argue, it's important not to overstate the differences between internal and external traces: the version of EM I'm developing here, following in particular in Andy Clark's wake, is aimed precisely at investigating a wide range of possibilities on these and other dimensions of variation. I distinguish two versions or "waves" of EM, differentiated partly by the way these dimensions are characterized. These waves are not ultimately incompatible, but they are distinct strands in the EM literature and within Clark's own work: as we'll see, he acknowledged "a potential tension" between them as long ago as 1998 (Clark 1998, p. 99; see section 4 below). Before introducing the two waves, let me reiterate the key commitment they share, tweaked and applied differently in each case. External symbol systems and other "cognitive artifacts" are not always simply commodities, for the use and profit of the active mind: rather, in certain circumstances, along with the brain and body that interact with them, they are (part of) the mind. For Clark, "it is our basic human nature to annex, exploit and incorporate nonbiological stuff deep into our mental profiles" (2003a, p. 198). The human mind is "leaky" both because it thus extends beyond the skin to co-opt external devices, technologies, and other people, and because our plastic brains naturally soak up labels, inner objects, and representational schemes, internalizing and incorporating such resources and often redeploying them in novel ways.

\subsection{EM and Interdisciplinarity: Historical Cognitive Science}

These general claims about the nature of the (extended) mind have been developed through striking examples, such as Otto with his notebook (Clark and Chalmers 1998, this volume). But concentration on the metaphysics of Otto's case, I'll suggest, underplays the ambition of the EM hypothesis, which can be better grasped by examining its far-reaching methodological dimensions. Perhaps, to study even mundane mental states and processes, we will need to look beyond the skin of cognizing organisms (Rowlands 1999, p. 8). Consequently, proponents suggest, EM motivates new perspectives on relations between the cognitive sciences, the social sciences, and the humanities disciplines. In a programmatic statement, Clark pinpoints "the single most important task" for "a science of the biotechnological mind" as the search for better understanding of "the range and variety of types of cognitive scaffolding, and the different ways in which non-biological scaffoldings can augment (or impair) performance 
on a task" (Clark 2002a, p. 29, my emphasis). Elsewhere he clarifies the dramatic implications of this agenda:

Much of what matters about human intelligence is hidden not in the brain, nor in the technology, but in the complex and iterated interactions and collaborations between the two. ... The study of these interaction spaces is not easy, and depends both on new multidisciplinary alliances and new forms of modelling and analysis. The pay-off, however, could be spectacular: nothing less than a new kind of cognitive scientific collaboration involving neuroscience, physiology, and social, cultural, and technological studies in about equal measure. (Clark 2001a, p. 154)

Such wild and whirling promises are not welcomed by EM's critics: Keith Butler, for example, argues that this kind of anti-individualism is "wrong headed," for

it tries to turn psychology into a kind of anthropology or sociology or ecology; and it just won't fit. There already are sciences whose topic of inquiry is the interpersonal and environmental. . . . There is no room for an expanded psychology, no motivation for it, and no need for it. (Butler 1998, p. 222)

In this chapter I aim to unpack the line of thought which takes us from EM to a methodological recommendation of strong interdisciplinarity ${ }^{3}$ for cognitive science. The path here is to display the methodological implications of EM at work in two historical case studies, rejecting any neat disciplinary division of intellectual labor. The EM framework is shown to be more than a mere philosophical injunction: the case studies operate within it, and their contributions to historical debates of independent interest are best understood in its terms. EM thus promises to be a potentially unifying gloss on and stimulus toward a wide array of research in diverse disciplines and subdisciplines which study those "cognitive and computational architectures whose bounds far exceed those of skin and skull," in which we "biotechnological hybrids" are primed to participate (Clark 2001b, p. 138).

But it's worth pointing out immediately that no imperialist agenda is necessary here: the possibility of an "expanded psychology" does not wipe out or negate all psychology's existing frameworks. First, EM is not a claim that cognitive processes are necessarily extended. Whole swathes of work on particular internal systems and processes can go on in relative independence of investigation into external processes, and any realistic proposal for active interdisciplinarity in the cognitive sciences is still likely to set mainstream experimental psychology and neuropsychology at the heart of the sciences of the mind. ${ }^{4}$ But without more careful, unprejudiced attention to the extraordinarily diverse array of research within psychology- 
across developmental, social, and personality psychology as well as cognitive psychology, and social-cognitive and systems neuroscience as well as cellular and molecular neurophysiology-we should not assume that these current cognitive sciences are wholly or primarily committed to a form of individualism that's in any substantial tension with EM. ${ }^{5}$

Yet two misreadings of this disclaimer, about the compatibility of EM with existing cognitive psychology of memory, need to be warded off. First, this doesn't mean that all those lines of research which do focus on internal systems and processes will remain untouched by EM (section 5 below). Second, we should reject the recent characterization of my inclusive and synthetic approach, with its recommendation that the EMinspired sciences of the interface be seen as complementing rather than replacing cognitive psychology, as "a non-revolutionary approach to embodied cognition" (Adams and Aizawa 2008, p. 179): this attempt to deflate and assimilate the current version of EM, as I seek to show in the remainder of this chapter, fails to recognize the novelty of a genuinely integrated interdisciplinary approach.

By developing the arguments for EM in the right way, Clark's distinctive and ambitious vision of interdisciplinary interaction can be elucidated, defended, and applied, and also refined and extended. Clark's (1997) own influential synthetic presentation of EM in Being There incorporated ideas from developmental psychology, robotics, human-computer interaction, dynamical systems approaches to cognition, complex-systems theories in biology and economics, and post-connectionist computational modeling. As he realized quickly, there was no good reason to stop there, and his inquiries have moved on to wearable, tangible, and ubiquitous computing, to telerobotics and neuroprosthetics, and to the future of software agents (Clark 2003a). But despite Clark's pleasure in novelty, he is also explicit about the fact that EM does not depend on or apply only to our couplings with new technologies. We are cyborgs by nature, and the human mind has never been "bound and restricted by the biological skin-bag . . . the ancient fortress of skin and skull" (Clark 2003a, pp. 4-5). Ambitiously reclaiming the language of human nature from narrower forms of evolutionary psychology, Clark argues that "our technologically enhanced minds are barely, if at all, tethered to the ancestral realm" or "constrained by the limits of the on-board apparatus that once fitted us to the good old Savannah" (2003a, p. 197; 2005b, p. 242; cf. Wheeler and Clark 2008). This opens up the cognitive sciences to history and culture, so that the EMinspired study of our "extended cognitive physiologies" can (and should) draw on and in turn enrich certain lines of research in historical disci- 
plines as well as in economics, sociology, science studies, and media theory.

It's in this spirit that my complementary studies in EM look backward. Since there's nothing particularly "posthuman" about EM-since, if we are cyborgs now, we always have been-there should be room for what I've called a "historical cognitive science" (Sutton 1998, 2000, 2002a; Richardson 2004, p. 23; Tribble 2006) to sit alongside work in cognitive anthropology (Hutchins 1995) and cognitive archaeology (Renfrew and Scarre 1999; Knappett 2005; Knappett and Malafouris 2008; Renfrew, Frith, and Malafouris 2009) as productive allies for EM. To see what might be gained in practice, I sketch here two examples of such historical work: Evelyn Tribble's study of the extended cognitive system of Shakespearean acting companies (Tribble 2005), and my own account of the 'arts of memory', the strange techniques inherited from the ancients that were popular in the medieval and Renaissance periods for internalizing elaborate architectures to aid recall and cognitive discipline (Sutton 2000). In each case, historical topics of entirely independent scholarly interest can be given a new twist by the EM framework: conversely, quite specific ideas in that framework are further explicated and illuminated in its applications. And as well as exemplifying historical cognitive science, both help me to describe and defend the second of two lines of thought behind the case for EM.

\subsection{Two Waves of EM Thinking}

Failure to pick up the existence of quite different agendas behind EM has led both critics and proponents into trouble. Most published discussion has focused on first-wave EM, so I start in section 2 by explaining why problems which some have thought apply to EM in general in fact apply only to its first wave. (I'm calling these "waves" partly because they don't really qualify as arguments for EM, and partly because the second flows from the first.) In this chapter I merely distinguish between first- and second-wave EM, and defend the conceptual priority and fruitfulness of the second wave; then toward the end of the chapter I suggest some ways of stretching the second wave, which might be natural extensions or which might turn out to require a distinct third framework.

First-wave EM is based on the parity principle (Clark and Chalmers 1998, reprinted in this volume): cognitive states and processes extend beyond the brain and into the (external) world when the relevant parts of the world function in the same way as do unquestionably cognitive processes in the head. If "exograms" act as engrams do, then for explanatory purposes they can be treated as engrams, the difference in their location being entirely 
superficial. Thus breaking down classical and individualist distinctions between brain, body, and world, we see that the object can be (part of) the subject, and that, as we've noted, things can have a cognitive life.

Second-wave EM is based on a complementarity principle: in extended cognitive systems, external states and processes need not mimic or replicate the formats, dynamics, or functions of inner states and processes. Rather, different components of the overall (enduring or temporary) system can play quite different roles and have different properties while coupling in collective and complementary contributions to flexible thinking and acting. So "exograms" can be radically unlike engrams even while co-opted for the same purposes, and these differences will often be the focus of complementarity-oriented explanations in the EM framework. We need both historical and developmental accounts of how our brains have just about managed "to make the world smart so that we can be dumb in peace" (Clark 1997, p. 180).

Although there is clear evidence for both these lines of thought in Clark's own work and in other recent EM literature, most critics have addressed parity alone. ${ }^{6}$ The two waves are not incompatible, but secondwave EM offers natural answers to a number of objections to EM which spring from parity considerations. But-although I won't labor the case here-complementarity too, I'll suggest, may have to be stretched considerably to deal with more deterritorialized investigations of shifting networks of heterogeneous components temporarily clustered or clumped together in contingent coalescence.

With these blunt statements of the two waves in place, I go on now to examine first-wave EM thinking. Along with other problems arising from the parity principle, at least as strictly interpreted, it threatens to undermine the methodological recommendations I've mentioned, and to render EM's connection with the search for "new multidisciplinary alliances" entirely mysterious. It will take recourse to the second wave to salvage the interdisciplinary agenda.

\section{First-Wave EM: Parity}

\subsection{The Parity Principle}

Clark and Chalmers introduced and defended EM by interpreting their examples in the light of a parity principle:

If, as we confront some task, a part of the world functions as a process which, were it done in the head, we would have no hesitation in accepting as part of the cognitive 
process, then that part of the world is . . part of the cognitive process. (Clark and Chalmers, this volume, p. 29; compare Clark, this volume, chap. 3, sec. 1)

This principle motivates the idea that Otto's notebook is itself playing a cognitive role when he uses it to get to MoMA. When extended from cognitive processes to cognitive states, it also then animates the stronger claim that the standing information in the notebook counts as cognitive even when it's not in use, because it functions in relevant respects just as do the standing, non-occurrent beliefs and memories in Inga's brain.

The parity principle stresses the functional isomorphism of inner and outer processes and states. It would be chauvinistic and unfair to treat Otto's notebook and Inga's brain differently just because one is external: and, claim Clark and Chalmers, since they play the same functional role in driving behavior in both cases, and when not in use are both still poised and available to play those same roles, there is no good reason at all to treat them differently. In particular, we can take it that Otto's notebook is as accessible, as reliable, and as transparent in use as is Inga's brain: though it can, under certain nonstandard circumstances (like theft and sleep), become inaccessible, the same holds for Inga's biological equipment.

It's this parity principle which gives EM its immediate metaphysical bite, enthusing sympathizers and infuriating critics. Parity is EM in critical mode, rejecting boundaries between brain, body, and world, undermining the easy assumption that the cognitive is inner and the outer is noncognitive. The "parity probe" was intended, Clark comments, "as a means of freeing ourselves from mere bio-chauvinistic prejudices," and "as a kind of veil of metabolic ignorance" which was "specifically meant to undermine any tendency to think that the shape of the (present day, human) inner processes sets some bar . . . on what should count as part of a genuinely cognitive process" (2005a, p. 2; 2008, p. 114). In John Haugeland's metaphor, the aim is to get "the whole rug smooth." We shouldn't, argues Haugeland, treat brain and body as clearly separable components joined at a well-defined psychophysical interface, nor can we slide "the hump in the rug" outward by identifying principled interfaces between body or sense organs and the physical world: instead, "we have to make it all lie flat" by denying that the mental is "categorically different" in kind from "anything bodily or worldly" (Haugeland 1998, pp. 228-229).

This urge toward parity also derives from considering studies of "densely coupled unfolding" (Clark 2005b, p. 234) in the dynamical and embodied cognitive sciences. Paradigm cases of continuous reciprocal causation show the rich real-time integration of neural, bodily, and worldly processes: the 
exploitation of continuous perceptual-motor feedback allows us to achieve complex and flexible action in rapidly changing environments (Beer 2000). In such cases, where the world can serve as its own representation, even if (contra Haugeland) inner and outer aspects of the coupled system can for certain purposes usefully be treated as distinct components (Grush 2003; Clark 2007), nonetheless the relevant dynamical parameters will often span body, brain, and world, rendering less significant any differences in the respective implementing mechanisms.

\subsection{Problems with Parity: Active Memory}

But exclusive focus on these cases, and on the case of Otto, where parity considerations do naturally apply, can cause trouble. The existing critical literature on EM has gone astray in ways which suggest that this first-wave line of thought at least needs clarification. Clark acknowledges that the original parity principle has been "subject to a persistent misreading" (2008, p. 114): in distinctive treatments which need attention on another occasion, he and Mike Wheeler have sought to restate, revise, and defend it (Wheeler, this volume). In contrast, here I suggest that we can move EM along both faithfully and fruitfully by downplaying parity and focusing instead on an alternative route to EM.

There are two stages to the initial dialectic here as we hone in on the first significant problem for parity. A number of individualists point out, first, as if in criticism of EM, that Otto has to use motor and visual processes which are not required by Inga (Adams and Aizawa 2001, p. 55; cf. Butler 1998, pp. 211-212, and the discussion in Chalmers 2008). This is to push unrealistically hard on the parity principle, as if EM is refuted if there are any differences between relevant intracranial and transcranial processes: so, further, discussing different ways of doing long multiplication, Adams and Aizawa think it relevant to point out that someone who does the computation without using any external aids does not use the same visual and motor processing as someone using pencil and paper to do the sum (2001, p. 59). Likewise, Bernecker offers as part of an "objection" to EM the point that "unlike a notebook, biological memory need not be charged, may get wet, and is immune to the Y2K bug" (Bernecker 2010, chap. 6).

Clark rightly responds in "Memento's Revenge" that EM does not require "that the processes in Otto and Inga are identical, or even similar, in terms of their detailed implementation" (Clark, this volume, chap. 3, sec. 3). The kinds of functional similarity which, under the parity principle, are to count as relevant are not to do with specific mechanisms, but with the 
functional poise of the information in question: its accessibility, availability, transparency in use, and so on, no matter what other differences there may be in the specific ways in which it enters into ongoing processing. There are, after all, as Clark points out, likely to be radically diverse causal processes involved in different kinds of inner process, too.

As Clark recognizes, however, the initial worry can be restated in more persuasive form (O'Brien 1998; Dartnall, 2004, 2005; Weiskopf, 2008). Even abstracting away from incidental details of mechanism and realization, even looking at functional poise alone, are not the format and the dynamics of biological and nonbiological representations and representational schemes just too different? We start to lose our grip on how to incorporate Merlin Donald's (1991, p. 315) points about typical differences between engrams and exograms into the EM story if we focus on parity alone. The storage and organization of information in Otto's notebook is, in Donald's terms, typically exogrammatic. Notably, information is stored there in discrete fashion, and representations in the notebook (linguistic or pictorial representations, for example) have no intrinsic dynamics or activity, are not intrinsically integrated with other stored information, and do no cognitive work in their standing or dispositional form. Representations in Inga's biological memory, in contrast, may well blend and interfere: according to connectionist accounts of memory, for example, non-occurrent standing representations, "stored" superpositionally in a single network's weight matrix, influence processing continually in a holistic fashion and are themselves subtly shaped by this ongoing history (McClelland and Rumelhart 1986). This dissimilarity, unlike the other superficial dissimilarity, matters.

But like Donald, Hutchins, Rowlands, and other post-connectionist theorists of the extended mind, Clark is of course well aware of such differences (Clark 1989, chap. 5; 1993, chap. 2). It's true, as he points out, that the existence of these differences is contingent, and that some or all of our biological memory systems might be, or might have been, less dynamic than radical connectionism suggests (Clark 2005a, pp. 5-7). It's also true, conversely, as I'll stress below, that not all external cognitive artifacts are as static and permanent as Donald suggests. So in some cases the homogenizing of inner and outer suggested by the parity principle will be salvageable.

Even where inner and outer resources are clearly heterogeneous in functionally relevant ways, where there really are significant differences in format and dynamics between engrams and exograms, application of parity may still be part of an EM-style explanation. In such cases, parity can operate alongside Clark and Chalmers's various criteria for treating 
objects as genuine parts of a cognitive system. These criteria of "glue and trust" (Clark, this volume, chap. 5) might include the requirements that the external resources should (when needed) be accessible, actually used, more or less automatically endorsed, and more or less reliable. Here I'm not defending any particular set of criteria. Rather, I note first that such criteria are, significantly, matters of degree: it's possible that, though they may clearly be satisfied on some occasions and clearly unmet on others, there will be many uncertain cases in between. This is, I suggest, not a cause for concern, as it might seem if your primary concern is establishing a single clear-cut mark of the mental: rather, the existence of a number of distinct dimensions on which particular cases can differ is the sign of a promising multidimensional space for doing EM-inspired cognitive science, by developing taxonomies or typologies of external resources in use, or of coupled systems (Poirier and Chicoisne 2006; Sutton 2006; Barnier et al. 2008; Wilson and Clark 2009). Second, notice that once any set of such criteria is brought in to adjudicate on particular cases, it's not the parity principle itself doing the real work: we're now seeing the existence of a range of possibilities, on a number of distinct dimensions, and we may rightly suspect that a more general and inclusive framework is needed, within which to locate many different kinds of case. ${ }^{7}$

\subsection{Problems with Parity: Individual Differences and Interdisciplinarity}

Such a framework-in my terms, a shift to a second-wave EM based on complementarity-is not in formal contradiction with the parity principle, for the relevant functional isomorphism postulated by parity can hold at a very abstract level of task analysis. But although the parity principle is technically loose enough to allow the parts of the world which combine in a particular cognitive process to be wildly heterogeneous, it at least does not encourage attention to the distinct features of the components in particular extended cognitive systems. Because parity downplays-or even collapses-differences between inner and outer resources, it is in some tension with the interdisciplinary dreams with which we started: when certain criteria are met, parity suggests, we shouldn't care if exograms rather than engrams are involved in the production of intelligent behavior. If Inga's brain and Otto's notebook are playing relevantly similar roles in driving what they each do, then there's no special point in studying the peculiar properties either of brains or of notebooks and other external media. There are two aspects to this concern, related to the individuals and the artifacts in question respectively. 
First, parity leaves no obvious space for investigating individual differences in relation to EM, because it asks us to focus on generic features of cognitive states and processes, whether in the world or in the head. Yet we often want to understand the specificities of particular embodied subjects: just why and how one system-such as a particular embodied agent of one kind or another-can move between a variety of different artifacts. Moving around in our idiosyncratic and complex sociotechnological worlds, each of us can decouple from and recouple with external resources of quite different kinds on a regular and continuing interactive basis. Mainstream psychology has long studied individual differences in the ways people approach various cognitive tasks without significant use of external resources. So, likewise, even in tasks which can involve extended looping and coupling cognition, we're all familiar with folk who aren't content or able to leave the information out there in the world. Developing Rodney Brooks's antirepresentationist arguments, Haugeland writes that "it would be silly, for most purposes, to try to keep track of what shelf everything in the refrigerator is currently on; if and when you want something, just look" (Haugeland 1998, p. 219). But we all know people who do typically upload such information into their onboard biological memories: such individual differences in the amount and style of reliance on external resources are often glaring in the ways people plan and engage in complex activities, such as writing an academic paper, shopping for a party, or chairing a department meeting. Do I memorize the train timetable in advance, or do I just turn up at the station and see? EM theorists have a great opportunity to work closely with cognitive, social, and personality psychologists to understand such differences, which are often not superficial.

So even if, as Clark (2007) suggests, EM sees embodied agents like ourselves are "essentially incomplete" in that we are deeply sculpted and continually transformed by plugging in to such wider networks, this in no way commits us to treating what we bring to the interface as a blank slate. We often want to understand whatever diachronic stability and continuity particular subjects exhibit. Critics like Keith Butler (1998, pp. 208-210) and Rick Grush (2003, pp. 79-81) have raised this problem against EM in general: but in fact it applies only to first-wave, parity-driven versions of the framework.

On the other side of the coin, parity also threatens to flatten out the important differences between cognitive artifacts. The particular nature of the external resources, it may seem, does not really matter: as long as the resources are appropriately accessible and so on, we wouldn't need 
media theory, history, or any other "social, cultural, and technological studies" in cognitive science. Further, parity fails to explain or motivate the interest shown by cognitive anthropologists, developmental psychologists, sociologists of science, and historians in the different effects which different cognitive artifacts may in turn have on our brains, behavior, affective life, and cognitive structure. The parity principle, in short, fails directly to suggest study of idiosyncratic or peculiar features of particular external symbol systems, or of particular ways of interfacing with them.

For these reasons, then, the parity principle is either wrong or incomplete as a motivation for EM. My suggestion that on its own, parity is in some tension with the interdisciplinary dreams with which we started, is supported by the fact that the critics of EM who focus on parity considerations have been puzzled at the way EM theorists actually do engage in detailed theoretical and empirical consideration of both the nature and the use of extremely specific kinds of exogram in diverse external symbol systems.

Discussing Merlin Donald's "theory of exograms as part of the human cognitive architecture," Adams and Aizawa note that Donald himself gives both rich accounts "of the development of all manner of external representations, including body decorating, grave decorating, sculpture, Stonehenge, hieroglyphics, cuneiform, maps, graphs, and musical scores," and careful analyses of the many "ways in which the processing of exograms differs from the processing of engrams" (Adams and Aizawa 2001, p. 58). They cite approvingly Donald's discussion of the different "properties of engrams and exograms" in relation to (for example) medium-dependence, capacity, and constraints on retrieval path (ibid., pp. 58-59).

But Adams and Aizawa (2001) find it bewildering that Donald still pursues the EM agenda while thus agreeing with them that internal and external states and processes differ on important dimensions regarding representational format and dynamics. This shows that Adams and Aizawa have misunderstood the dialectic here, because they see EM as resting on parity considerations alone. Since Donald's framework is in tension with (or at least downplays) parity in stressing various dimensions of difference between exograms and engrams, Adams and Aizawa assume that it is thereby in tension with EM, and that Donald should consequently reject $\mathrm{EM}$ and embrace their conclusion that "there can be no cognitive science of transcorporeal processes" (2001, p. 58).

Yet, as we'll see in the next section, it's precisely this kind of investigation of the variety of cognitive interfaces, and the many dimensions on which differing inner and outer resources are unequal, which characterizes 
detailed and sophisticated work across the disciplines by writers who also defend EM and distributed cognition.

But then the humps in Haugeland's rug will multiply, rather than disappear. I noted above that the motivation of the flattening-out urge which drives first-wave EM is primarily critical. Immediately before his rug analogy, Haugeland has told us that "the idea is not to wipe out all distinctions and homogenize everything on general principles, but rather to call certain very familiar divisions into question" (1998, p. 228). The apparent shift in emphasis across these two passages is symptomatic of the more general dual movement I'm identifying in the EM literature, which oscillates between first breaking down confidence in entrenched boundaries and then, once distinctions have been exchanged for networks, reinstating heterogeneity. This dual pull is natural within EM thinking, and not a vitiating tension: rendering it explicit may help move the debates along. And after urging us to "make it all lie flat," Haugeland shows that he's aware of the tension by urging us not to read this advice "in a way that washes out all distinctions [between mind, body, and world], rendering the three terms synonymous" $(1998$, p. 230$) .{ }^{8}$ Since that's what the parity principle seemed on first reading to suggest, that first reading needs to be clarified or supplemented.

So, despite Adams and Aizawa's puzzlement, there must at least be an alternative motivation-or even justification-for fully interdisciplinary research from within the general EM framework. Before sketching such an alternative-second-wave EM, based on "complementarity"-let's illustrate it with a case study.

\section{Cognition in the Globe}

Evelyn Tribble's (2005) study "Distributing Cognition in the Globe" applies EM surprisingly directly to an existing historical puzzle. Inspired in particular by Hutchins's (1995) study of navigation, Cognition in the Wild, this impressive project is the most successful and intriguing historical application yet of EM-and the form of EM hypothesis which Tribble shows off in strongly interdisciplinary action, I'll suggest, is clearly a second-wave version.

One of many striking differences between modern Western theater and the repertory system of Shakespearean England (in the late Elizabethan and early Jacobean periods, either side of the year 1600) is that, as Tribble puts it, the (exclusively male) actors then performed in the Globe and other theaters "a staggering number of plays ... . with relatively infrequent 
repetition, and with the additional demands of putting on a new play roughly every fortnight" (2005, pp. 135-136). Between 1594 and 1597, for example, a leading player such as Edward Alleyn "had to secure and retain command of about seventy-one different roles, of which number fifty-two or fifty-three were newly learned" (Beckerman 1962, p. 9). Yet the actors did not have the full texts of these plays. How did the actors cope, and how did the companies rehearse and perform so many different plays under such pressures?

Studies of actors' memory in cognitive psychology (Noice and Noice 1997) can't fully answer these questions, because as Tribble demonstrates their models of acting cognition rely on quite different modern assumptions (about "character" and "subtext") and practices (such as long rehearsal periods, and few new plays). And existing scholarly explanations of early modern theater practices have gone, Tribble shows, in two mistaken directions. Either, it's been suggested, an actor rehearsed privately within a routinized formula, by which he covered every performance by playing roughly the same part, learning in "parrot fashion"; or an authoritative individual (Shakespeare himself?) must have been constantly present at rehearsal, like a modern director, to explain and install every aspect of each performance. Both views neglect the active cognitive role of the larger systems within which actors worked:

the nature of the playing system, and particularly of the mnemonic demands that the repertory system made upon its participants, has been consistently misunderstood because of a tendency to view cognition as individual rather than social and therefore to imagine the workings of complex group systems in mechanistic terms. (Tribble 2005, p. 135)

In contrast, Tribble offers a sustained reinterpretation of the historical evidence to show how diverse tools, practices, and aspects of the social and institutional form of the early modern theatrical system together "form elements of a cognitive structure that, in constraining and limiting, also enables an extraordinary level of achievement" (2005, p. 142). As in the expert navigational cognition described by Hutchins, so in the Globe physical architecture, artifacts, social structure, and the characteristics of the plays themselves combine to support the collective success of the company in performance.

It's not just that the stage space itself operated in conjunction with simple conventions as a cognitive map for sequencing the play's action. The vehicles supporting the actors' memory capacities included a diverse array of distinctive artifacts. Tribble focuses as well on early modern cue- 
scripts or "sides," and on some large documents called "plots" which were probably hung on walls. Both forms of surviving evidence puzzle scholars because the information they contain seems "impossibly scanty," quite inadequate by our standards. The cue-scripts contained only the barest of cues: instead of having copies of a whole play, actors were given only minimal information in textual form, and had instead to rely on hearing cues wrapped into the structure of the plays in performance. The cryptic "plots," in turn, which have seemed "maddeningly incomplete" to modern scholars, were concerned primarily with only entrances (but not exits) and scene division: if considered as compressed encodings of entire performances, they are failures. But Tribble, inspired by Hutchins, sees them instead as computational devices within extended cognitive systems, taking these two-dimensional renderings of the complex spatiotemporal paths of a performance to work in their immediate action-guiding context just because of the paucity and resulting clarity of the explicit information they held. As with the models and representations used in children's play, or in the complex design cognition of modern adults, the success of these various tools in supporting the actors' fluent performance may have depended, "to a certain extent, on actively keeping the level of nonessential detail quite low" (Clark 2005b, p. 237). Roughly, the Globe's artifacts worked to get the actors to the right place at the right time for further local environmental alterations (such as a particular line or event on stage) to call forth spontaneously the required specific behavior (cf. Clark 1997, p. 76, on Hutchins).

So before we even consider the mnemonic and action-guiding nature of the plays themselves, or the broader hierarchical social system of the playing companies, we can see that a wide array of factors conspire to solve the various coordination and memory problems facing the company. Contrary to any default assumption that the overall play is controlled by a single plan in the mind of Shakespeare as writer or Shakespeare as director, or by a single authoritative script, in fact the sequences of actions and interactions which collectively constitute the performance "need not be explicitly represented anywhere" (Clark 1997, p. 77, on Hutchins).

Though this isn't yet meant as a full explanation of the historical phenomena, Tribble's work dramatically remolds the issues so that they no longer look like problems about the capacity and limits of the individuals' memories. The actors' skill was not only in semantic memory for their lines or characters but also in their procedural memory or know-how, in their mastery of the cognitive resources of their highly structured dramatic environment. We want now to ask more about the interface between 
actors, props, plots, stage space, verse, and so on. We can follow Hutchins again (for example) in simultaneously examining the computational and social embedding of novices within the overall system: in the case of the Globe, apprentices initially played minor (theatrical and computational) parts in a smoothly functioning whole, and then might understand and internalize the appropriate actions and skills only after they were already performing them (Tribble 2005, pp. 153-155; Hutchins 1995, p. 224).

Tribble's careful analysis of cognition in the Globe demonstrates the practical value of the extended mind framework for offering new perspectives on cross-disciplinary topics of independent importance. It helps us to identify and undermine the temptation, common in cognitive-scientific investigation of complex collective action in specific cultural contexts, to think that some single or determinate component of the overall system (such as the cue-scripts, the plots, or the individual actors) must have fully encoded "the play" in order for a successful performance to emerge in practice. Further, it thus exemplifies the shift from first- to second-wave EM. Like the process of navigation described by Hutchins, the case of early modern acting differs from the case of Otto in an important respect: whereas Otto's notebook acts directly as an equal substitute for his brain, the cue-scripts and plots which (like Hutchins's nautical slide rules, charts, and so on) were elements in an extraordinary complex system of distributed social cognition are nothing like the internal resources brought to bear by the individual actors (or navigators). There's no sense in which the various nonhuman artifacts which scaffold successful performance have to be doing the same thing as the individual participants are, or even storing the same information as might have been stored in their individual brains: to quote Clark's apt account of Hutchins's case study again, "the computational power and expertise is spread across a heterogeneous assembly of brains, bodies, artifacts, and other external structures" (Clark 1997, p. 77, my emphasis).

\section{Second-Wave EM: Complementarity}

In addition to the parity principle, Clark has also stressed a different aspect of the case for $\mathrm{EM}$, one which is more sensitive to the "essential causal dynamics" (Adams and Aizawa 2001, p. 44) of inner and outer processes, respectively. In his response to Gerard O'Brien's (1998) critique, Clark defends this second line of argument for EM, noting that although it is formally compatible with parity, there is indeed "a potential tension" between them (Clark 1998, p. 99). This "more interesting and plausible 
argument" for EM turns on "the way external elements may play a role different from, but complementary to, the inner ones": in thus stressing "complementarity," we should see the functional isomorphism required by the parity principle as "at most part of a sufficient condition for cognitive extension, rather than a necessary feature" (Clark 1998, p. 99).

The parity principle, then, is better seen as "an informal test" (Clark, this volume, chap. 3, sec. 3) or temporary indicator of cognitive extension, a place-holder for fuller, more inclusive sciences of the interface. Biological and nonbiological resources, in certain circumstances, work together, coalescing into integrated larger cognitive systems. As Clark writes in a key passage, the external resources on which cognition sometimes leans are best seen as alien but complementary to the brain's style of storage and computation.

The brain need not waste its time replicating such capacities. Rather, it must learn to interface with the external media in ways that maximally exploit their particular virtues. (Clark 1997, p. 220)

With this complementarity principle, as we might call it, ${ }^{9}$ we return connectionism to the heart of the case for EM. It's just because isolated items aren't stored atomically in the brain that our relatively vulnerable biological memories are supplemented by more stable external scaffolding. Brains like ours need media, objects, and other people to function fully as minds. Seeing the brain as a leaky associative engine, its contents flickering and unstable rather than mirroring the world in full, forces attention to our reliance on external representations in the technological and cultural wild. The classical search for the engram (as an enduring discrete item stored at a fixed address) fell foul of the holistic and dynamic nature of representation in the brain: biological traces are typically integrative, active, and reconstructive, but in using them we hook up with more enduring and transmissible exograms, mostly of our own making, which supplement and extend our powers. According to this second-wave EM,

The argument for the extended mind thus turns primarily on the way disparate inner and outer components may co-operate so as to yield integrated larger systems capable of supporting various (often quite advanced) forms of adaptive success. (Clark 1998, p. 99)

The complementarity principle explains why, to the critics' dismay (section 2 above), an EM theorist would catalog differences between the properties of engrams and exograms: "unlike the constantly-moving contents of biological working memory, the products of thinking, when reformatted 
exogrammatically, could be frozen in time, held up to scrutiny at some future date, altered and re-entered into storage" (Donald 1991, p. 316). The EM perspective offers a typology or framework in which many different relations (and kinds of relations) between inner and outer resources can be understood. Cases like Otto with his notebook, which are transparent examples of parity in that a single external resource is doing just what could otherwise be done in the head, can be seen as special cases of complementarity.

If complementarity thus subsumes and takes precedence over parity, it's clear that EM as a general thesis need say nothing about exactly what kinds of formats and dynamics there must be inside and outside the skin. Different kinds will permit and encourage quite different kinds of interaction and coupling and thus different kinds and degrees of extendedness. EM, thus understood, is more an invitation to give detailed attention to these differences in specific contexts and case studies than a fixed new metaphysics of mind. So its second wave both encourages and makes sense of the interdisciplinary agenda I described in section 1 and exemplified with Tribble's case study. Such an agenda, of course, may bring problems of its own. In section 6, after we've looked at another historical example, I'll briefly consider problems about the nature of explanation in this form of EM. But I want to close this section with two different challenges for second-wave, complementarity-based EM, without here needing to decide whether they will turn out to be manageable within its framework, or will require sufficient amendment to justify thinking in terms of a third wave.

First, it's important to resist a tendency within second-wave EM still to treat the inside and the outside as distinct realms with fixed properties. Merlin Donald's tabulation of the different characteristics of engrams and exograms does deal successfully with the problem critics raised for parity, about the active, reconstructive, and context-dependent nature of biological memory, in contrast with the passive, stable, medium- and contextindependent nature of external symbol systems. But, to repeat, not all exograms are as discrete and as fixed as is the information about the museum recorded in Otto's notebook: external representational systems need not be permanent, of unlimited capacity, translatable across media, or endlessly reformattable as Donald's typology suggests (see also Sutton 2008, 2009). The words and sentences in Otto's notebook, for example, might be replaced by some much more dynamic new-media system incorporating a range of sensory modalities, which is continually updating or appropriately reconfiguring in ways which (we could imagine) still met the criteria of accessibility, direct availability, and automatic trusting 
endorsement; in a further extension, such a system might come to highlight information or options aligned with Otto's moods or emotions. Think of the various ways in which other people enter our external memory fields, with their dynamic engrams potentially our exograms (Wegner 1987; Wegner, Erber, and Raymond 1991). Another as yet merely fictional example is the personalized book A Young Lady's Illustrated Primer in Neal Stephenson's (1995) novel The Diamond Age, which bonds and couples with a particular little girl, initially through simple imprinting but soon through a brilliantly imagined range of interactive technologies, which include both dynamic databases and live actors-at-a-distance. More generally, not all systems of exograms are meant to be permanent or endlessly transmissible: and not all such systems which are intended to endure actually do so (Kwint 1999). Whether by design or not, medium and message are often not as independent as in Donald's scheme: the degree of contextdependence itself depends on the context. In relation to permanence, medium-dependence, ease of reformatting, and all the other dimensions of Donald's picture, nothing intrinsic to the second-wave complementarity framework imposes such stark gaps between the natural and the artificial: so genuine interdisciplinary inquiry should not assume them.

Second and conversely, we need to see just how naturally complementarity can make sense of a further strand of EM-inspired research: the investigation into ways in which integration into larger cognitive systems may alter even the inner parts of those larger systems. As Clark has long argued, "cognitive technologies" don't have to be external: among the many resources we use to think about (for example) the past, the abstract, and the absent are a range of internalized representations and symbol systems, which we learn (historically and developmentally) to manage with both idiosyncratic and culturally specified techniques. The first step in this line of thought, taking us into a second historical case study, is to recognize that it's not that the same basic inner resources are brought to the interface with all these different media and symbolic technologies and left untouched. If that were the case, then cognitive science could remain the study of individuals, interacting variously with different external artifacts. Instead, in turn, such interfacing is often inherently transformative. Consider Clark's account of language (Clark 1997, 2005c; see also Millikan 2001; Sutton 2002b; Wheeler 2004): drawing on developmental studies of inner speech and representational redescription, Clark sees language as not primarily a tool for the communication of fully formed thoughts, but as providing us with a code which "minimizes contextuality" and is itself the ultimate cognitive artifact. 
By "freezing" our own thoughts in the memorable, context-resistant, modalitytranscending format of a sentence, we thus create a special kind of mental objectan object that is amenable to scrutiny from multiple cognitive angles, is not doomed to alter or change every time we are exposed to new inputs or information, and fixes the ideas at a high level of abstraction from the idiosyncratic details of their proximal origins in sensory input. (Clark 1997, p. 210)

Like other internalized schemes, language here is itself a kind of prosthesis, an imported aid which allows for higher-order dynamics of selfcontrol and mind control that can, as we'll see, be cognitive and affective at once. To continue pushing at the edges of this second-wave, complementarity-inspired form of EM, we can move on to our second case study in historical cognitive science, EM-style.

\section{The Arts of Memory and the Civilizing Process}

The remarkable medieval and Renaissance memory practices have been richly described by such wonderfully interdisciplinary modern scholars as Frances Yates and Mary Carruthers, whose works should be consulted for historical detail of specific mnemonic practices and writings (Yates 1966; Carruthers 1990). Although the broad techniques in question have survived from the ancient world to the present and are still studied in contemporary applied cognitive psychology (Moè and de Beni 2005), we can find in their heyday an intriguing case study in how cognitive artifacts were internalized in an alien moral, social, and theoretical context.

The historical distance afforded by this kind of exercise is vital, because it's often harder to see the mutual entanglings and contaminations operating between brains, technologies, and culture in the present. Among other things, this work is a first step toward answering John Haugeland's challenge to EM theorists to care more for the public norms, communal practices, and moral dimensions of our relations with artifacts (Haugeland 2002). Haugeland complains that Clark's approach is impoverished because it draws so much on work involving robots, infants, and other animals, none of which is a full member of a human community, with the fullblown histories and traditions which attach to complex public norms. So any help which EM offers in understanding such culturally embedded practices as these old methods of managing memory and imposing cognitive discipline might help us to see some of the "roots of norm-hungriness" (Clark 2002b) in our ability to interiorize relatively stable forms of culturally sanctioned scaffolding in the quest for self-mastery. ${ }^{10}$ 
Each adept gradually constructed an artificial memory, a set of locations or places (in a memory palace or theater, an ark or an alphabet or an angel, a grid or a bestiary). This permanent set of locations could then be used to hold the images or other items to be remembered. Each representation encoded must be independent of all others, with each content mapping individually on to its place: that's why strict division of material was required, to keep items distinct. This atomistic storage system then allowed the remembering subject to move around the memory locations at will, extracting or manipulating items from their arbitrary addresses. Despite the apparent doubling of effort required to remember both the locations and then the specific items to be remembered, the system was both economical and flexible, for once the virtual architecture was securely internalized, it could be used and reused at will. The adept's mind had become a random access memory (Carruthers 1990, p. 7, 1998, p. 16).

The key, then, was the static nature of the items, whether they were bizarre images placed on memory plinths or verbal cues written onto memory tablets. Mistakes were due not to distortion in recall, but to failure to render images distinct enough at the time of encoding. After successful encoding, items are context independent, to be inspected, recombined, and transformed again only under deliberate executive control. So the system has no intrinsic dynamics, and semantic stability is maintained across contexts.

What does this have to do with the extended mind hypothesis? These Renaissance scholars were not hooking up with any literally external technologies: they were specifically refusing to use the world as its best model, instead laboriously soaking up whole baroque memory edifices. Of course there's a complicated narrative to trace here about the history of books and the spread of print media, which would address changing historical relations between these cognitive practices and the available external artifacts for recording and transmitting information. But taking EM seriously, I suggest, means that we treat such architectures, systems, and practices as both cognitive and extended whether or not they happen to be outside the skin. They are cognitive even though they are not, in a straightforwardly ancestral way, natural and biological; and they are extended even though they are not literally external. The cognitive skills which individuals roam round with, more or less successfully, have histories which are just as much cultural and developmental as biological.

In particular, this example shows starkly how cognitive practices can be tangled in with communal norms and moral requirements. These 
techniques were explicitly driven by awareness that what scholars called "natural memory" had built-in tendencies to confusion. Artifice was needed because of the corrupt nature of embodied humanity, where one effect of the Fall was loss of control over the personal past. Especially by the Renaissance period, the eclectic default humoralist physiology offered little room for unaided inner stability. ${ }^{11}$ So the natural dynamics, the "disorderly floating" of ordinary memory had to be suppressed. Even the use of strikingly affective images, bloody and violent, in the memory palaces, was aimed at neutralizing indiscipline, containing emotional items in their places. These schemes thus operated on a picture of the relations between the natural and the artificial, the biological and the virtual, which is very like the post-connectionist account of the profound differences between engrams and exograms through which I characterized second-wave EM thinking, based on complementarity, above: and this perspective guided my own earlier take on the arts of memory, which I now think needs some amendment.

In that earlier work, I saw the localist style of representation in the memory arts, with its built-in fantasy of totally voluntary memory, as a wishful stabilizing of confusion from above. The techniques were meant to supplement and strengthen, or more often to supplant and bypass entirely, the mixture and blending which was natural to the roving animal spirits as they ceaselessly constructed their overlapping patterns of flow in the spongy brain. Cognitive discipline was an achievement, to be carefully worked at and guaranteed by the interiorizing of these sanctioned supplements. Escaping the murky forests of natural memory, the adept resists the crowding and interfering of traces in the brain, and traps all intensity in his memory rooms.

I still think it clear that the arts of memory were a moral quest, to ward off the intrusion of unwanted thoughts, to undertake the disciplined purging of what Saint Bernard called "filthy traces" from the past (Coleman 1992, pp. 182-191). But now I think this analysis was taken in by the rigidity of the historical practitioners' own dichotomy between natural and artificial memory. I saw the quest for control over items in memory, guaranteed by separating data from process, memory from executive self, as the external and artificial imposition of order by reason or will on the true and naturally confused memory system of fleeting animal spirits. So-in rather primitivist fashion-I saw the arts of memory as the cognitive wing of a heavily moralized civilizing process: by freezing the contents of memory, and locking them into separate rooms for later extraction, monks and scholars sought to tame and recalibrate their minds, in a 
retreat from multiplicity, disturbance, and embodiment. In a similar vein, in his recent reading of the same techniques Paul Ricoeur describes the ars memoriae as "an outrageous denial of forgetfulness and . . . of the weaknesses inherent in both the preservation of traces and their evocation" (2004, p. 66).

But now I want to develop further themes from Clark to question this kind of reading. In recent work on "self-scaffolding," Clark surveys various forms of "virtuoso artificial self-manipulation" available to symbol-users like us. By finding tags, labels, or images for higher-order patterns in our own thought and action, we create "a kind of affect-dampening layer of insulation," which allows us "to productively dampen and control (but not counter-productively destroy altogether) the fluidity and contextsensitivity of biologically basic forms of neural representation" (Clark 2005c, pp. 263-264). These abilities "to vehicle our thoughts" in language and in other representational formats (Clark 2003b) are entirely continuous with the other, more direct ways in which we actively restructure our external environment (cf. Dennett 2000). Clark describes a general need for "anchoring" in terms highly reminiscent of the medieval and Renaissance monks and scholars: we collectively and individually devise "cognitive strategies (which may be more or less indirect and baroque)" to address "the problem of stabilization," the need to discipline our "mental spaces in ways that tame (though never eradicate) those biologically more 'natu-

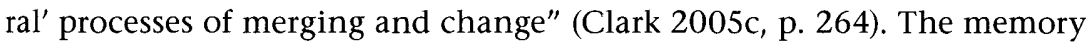
artists' active training in memory and meditation, from this perspective, starts to look more like a particularly explicit attempt to develop the ongoing capacity to treat memories and other items as themselves objects for thought, to buffer or influence their affective impact, and (again in Clark's terms) to "drive, sculpt and discipline the internal representational regime" (ibid.).

I don't want to deny the historical excesses of inebriated rationalism in the Renaissance memory arts, as noted in my prior analysis and by Ricoeur: but Clark's take on cognitive discipline might also help us to see even such ostentatious strategies of memory control as natural for human cyborgs like us. Despite the ease with which both Clark and I have contrasted, in our different contexts, the fluid biological memory with the more rigid artificial system, we shouldn't take this profound dichotomy between engrams and exograms as the end of the story. It's not quite right to treat the true, or natural memory as that given by the brain alone, whether by humoral nervous fluids or by post-connectionist neural networks. Why should we treat the internal prosthesis provided by the memory palaces 
and their internalized exograms as genuinely external to the mind? Are culture, artifice, and moral practice optional extras, merely dispensable surrogates which ride on top of the brain's own unchanged tendencies? Or are they instead in one form or another inevitable, structuring supplements which construct and maintain the biological processes that they simultaneously and deeply transform?

In contrast to later moral physiologists who simply denied the productive cognitive role of mixture and blending in the brain, these earlier memory practitioners took it very seriously. ${ }^{12}$ That's why they were so sensitive to the need for artifices and prostheses-in this case, internalized prostheses-creating secure locations, virtual nooks and clear unswampy corners of the memory, secret angles of the mind in which they hoped to find what and only what they had deliberately put there. Of course the quest is imperfect: as Hamlet discovered, despite his promise to the ghost, "baser matter" doesn't just disappear, and the personal past doesn't always flatten out. But it's not as if we can avoid leaning on artificial systems. Recalibration is ongoing, as we alter our own cognitive machinery by exploiting and importing whatever tools and labels we can. The memory artists' skillful use of a manageable and reliable set of cognitive artifacts was an unusually developed, culturally anchored way to deal with contextuality. The civilizing process, thus understood, includes the tidying of our own brains as well as of our behavior, and it isn't really optional.

This slightly shifted picture of these weird old practices should also have some historical benefit: in particular, it allows us better to incorporate Mary Carruthers's rich and persuasive work on the meditative aspects of mnemotechnics as a skillful "craft of thought" (Carruthers 1990, 1998). Where previously we might have seen a "deadly infatuation" with the exercise of sovereign choice after an "original denial" of "the constraints of traces" (Ricoeur 2004, p. 66), by putting Carruthers's revisionary history together with Clark's version of EM we can reinstate a sense of the practical cognitive and emotional labor, and the riskiness of the quest for wisdom in the "things" and the "devices" of this "architecture for thinking" (Carruthers 1998, pp. 7-35). ${ }^{13}$ Just as in offloading both information and procedures into external technologies and social systems we thereby reconfigure our cognitive tasks and profiles, so in constructing elaborate inner machines for sedimenting and working with affectively laden images and thoughts, the memory artists gradually developed different cognitive skills. Discussing the basis of composition and digression in the memorybased arts of rhetoric, Carruthers and Ziolkowski describe-in terms which might put us in mind both of Hutchins and of Tribble-the memory 
expert's ability spontaneously to shuffle and reorder, to gather up materials and then find the way again: once the inner architecture is securely founded and its navigational principles well practiced, then "I can always be sure of 'where I am' in the composition-not in the manner of a parrot (which, reciting mindlessly, never knows 'where' it is) but in the manner of an experienced harbor pilot recalling landmarks" (Carruthers and Ziolkowski 2002, p. 5).

Many "high-level," "representation-hungry," and "decoupled" cognitive processes-whether thinking about intimate aspects of the long-gone personal past, or predicting the effects of a shift in foreign policy, or recollecting the ways to salvation through the gospels-occur in the absence of actual or possible immediate external stimulation. But, as Clark (2005b) argues, this does not mean that they are decontextualized and disembodied. For just as in other cases we create "surrogate situations" in the external world so as to amplify cognition and "direct and distribute attention in new ways," so-like the dead adepts of the memory arts-we can also use culturally sculpted internalized surrogates.

Like Clark's treatment of language (section 4), this is starting to push the second wave's nice dichotomy between fluid inner engrams and stable outer exograms pretty far: as Hutchins argues, "it is not that some content is copied from the outside world into some internal storage medium . . . what used to look like internalization now appears as a gradual propagation of organized functional properties across a set of malleable media" (1995, p. 312). ${ }^{14}$ If there is to be a distinct third wave of EM, it might be a deterritorialized cognitive science which deals with the propagation of deformed and reformatted representations, and which dissolves individuals into peculiar loci of coordination and coalescence among multiple structured media (Hutchins 1995, p. 316; Sperber 1996, pp. 57-63; Suchman 1998; Johnston 2002, pp. 481-482; Clark 2003a, pp. 130-142, 2004b, pp. 177-180; Mackenzie 2004). Without assuming distinct inner and outer realms of engrams and exograms, the natural and the artificial, each with its own proprietary characteristics, this third wave would analyze these boundaries as hard-won and fragile developmental and cultural achievements, always open to renegotiation.

\section{Conclusion: A Note on Explanation}

Even the basic second-wave complementary route to EM, of course, has its costs. As critics and friends of EM alike have realized, the extended cognitive architectures it would encompass include a daunting array of the 
social and technological systems with which embodied brains can couple. What would cognitive science be like, how could it continue, if its objects include notebooks, sketchpads, and tattoos as well as embodied brains? Systems of brains coupled with cognitive tools, computing devices, or memory aids, complain Adams and Aizawa, "would seem to form such a motley collection that they will not form the basis for any significant scientific theorizing" (2001, p. 63). EM thus threatens to thwart cognitive science's connected quests for natural kinds and for disciplinary identity. If-to sample the relevant literature-other people, scrabble tiles, theater architecture, cocktail glasses, slide rules, incised sticks, shells, languages, moral norms, knots, codes, maps, diagrams, fingers, monuments, software devices, rituals, rhythms and rhymes, and roads can count as part of the legitimate subject matter of the sciences of mind, isn't EM obviously absurd? The fear is that EM would leave cognitive science paralyzed, in the same way-and for the same reason-that Tooby and Cosmides mock "mainstream sociocultural anthropology" for being in "a situation resembling some nightmarish story Borges might have written, where scientists are condemned by their unexamined assumptions to study the nature of mirrors only by cataloguing and investigating everything that mirrors can reflect" (Tooby and Cosmides 1992, p. 42).

In conclusion, I want to underline the two responses to this worry that Clark offers in section 3 of "Memento's Revenge" (this volume, chap. 3). First, we shouldn't work with an overly restricted or puritanical notion of scientific explanation: nonpredictive narrative explanations are common enough in the natural and social sciences of many complex systems, including branches of history, geography, geology, evolutionary biology, and meteorology. ${ }^{15}$ This doesn't inevitably diminish these frameworks' rigor, whereas premature quests for lawlike regularities might: more particularized illustrative studies may just be the best way for us to get what Paul Churchland has called "objective knowledge of a highly idiosyncratic reality" (1996, p. 306). Of course, it will sometimes be fruitful artificially to simplify the hopeless multiplicity we're faced with in studying remembering, acting, thinking, interacting, feeling, talking, imagining, perceiving, planning and so on. But as I've argued before, amid the Kuhnian "normal science" of the modern cognitive sciences and neurosciences, with their vast apparatus and institutions, it might also be rational sometimes for some to indulge an "untidy preference for proliferation over prudence in difficult domains" (Sutton 1998, p. 3, 2004, p. 190).

And, second, we shouldn't rule out in advance the possibility that in fact there may be higher-level accounts which do find commonalities or 
patterns across the alleged "unscientific motley of capacities" (Adams and Aizawa 2001, p. 62) exhibited by extended hybrid minds. On this point we'll just have to wait and see. ${ }^{16}$ Just as Clark has been advancing his own optimistic vision of sciences of the interface, investigating "varied, multiplex, interlocking, and criss-crossing causal mechanisms" over "a wide variety of mechanistic bases" (this volume, chap. 3 , sec. 7) by exploring new cognitive technologies, so I hope the reverse-angle case studies sketched in this chapter have hinted at the patterns and possibilities which might emerge in historical cognitive sciences. At the very least, second-wave EM can thus tap and in turn influence the enormous and diverse scholarship on memory, perception, emotion, and so on in humanities disciplines, to see what might happen if we try to study cognition scientifically and culturally at once.

\section{Acknowledgments}

Special thanks to Richard Menary for his work as editor, and for organizing wonderful conferences on the extended mind at the University of Hertfordshire, where I first tried out some of these ideas. Later versions were presented at the annual conference of the Australasian Association for History, Philosophy, and Social Studies of Science in 2002, at the joint International/Australasian Cognitive Science conference in Sydney in 2003 , and at a reading group and workshop on memory and embodied cognition at Macquarie University in 2004. Many thanks to these audiences, to Andy Clark and Lyn Tribble for their enthusiasm and help, and for various contributions and support to Samir Chopra, Hugh Clapin, Stephen Cowley, Tom Evans, Adam Holland, Eve Keller, Doris McIlwain, Andrew Murphie, Gerard O'Brien, Mark Rowlands, David Spurrett, Tony Stone, Maria Trochatos, Mike Wheeler, and Carl Windhorst. I'm particularly grateful to Tim Bayne and the late Terry Dartnall, who both wrote detailed comments on an earlier draft: I've tried to respond to all their queries and suggestions. For discussion on the arts of memory over the years, thanks in particular to Ed Cooke, Jim Franklin, Jamie Kassler, Doris McIlwain, and Gail Kern Paster.

\section{Notes}

1. I use the label "EM" for brevity throughout, as if this family of ideas were unified. This chapter is an exposition and development specifically of Andy Clark's version, although it hones in on distinct strands of his account of EM. But I treat 
the following labels from the philosophical literature as closely related: active externalism (Clark and Chalmers 1998, reprinted in this volume), vehicle externalism (Hurley 1998; Rowlands 2003), locational externalism (Wilson 2004), environmentalism (Rowlands 1999), systemic anti-individualism (Butler 1998), and transcranialism (Adams and Aizawa 2001). My take on EM, based on the "complementarity" rather than the "parity" of inner and outer resources, brings it closer to the related theory of "distributed cognition" (Hutchins 1995; Kirsh 2006; Rogers 2006), which arose independently in cognitive anthropology.

2. Despite its relevance and promise, I don't in this essay consider the "nonsymbolic environment," which some proponents of EM include (Haugeland 1998; Wilson 2004, pp. 192-196). So readers who come to EM from science studies and actor-network theory, or HCI (human-computer interaction) and CSCW (computer-supported cooperative work), or whose interest is in material agency and posthuman technics, will likely find (on this point as on others) that the underlaboring, ground-clearing work I attempt in this chapter is disappointingly slow, as I'm still talking with cognitivists and trying to convert individualists: but for some initial suggestions see Sutton 2008. One aim of a third-wave EM might be to incorporate the best work of such theorists from outside the cognitive sciences, who often, as Clark notes (this volume, chap. 3, conclusion) regard EM "as patently true": see my brief remarks at the end of section 5 .

3. For present purposes we can understand "interdisciplinarity" by thinking of a spectrum from the easier mere juxtaposition of disconnected theoretical and empirical frameworks, toward more integrated novel projects and approaches which genuinely fuse disciplines and methods. Rogers, Scaife, and Rizzo (2005), in an important consideration of relevant methodological questions in the context of EM-related research, call the former "multidisciplinarity" and the latter "interdisciplinarity": cf. von Eckardt (2001). I outline a position toward the stronger end of such a spectrum in relation to memory research in Sutton 2004.

4. This makes some objections to the project look misplaced. Rick Grush, in a fine critique of John Haugeland's version of EM, worries that, if Haugeland is right, then "cognitive neuroscience as a discipline would be somewhat ill-formed, for it presumes to be studying perception, cognition, and the like, by focusing on neurophysiology. But if it does not make sense to treat the brain as a conceptually autonomous component, then cognitive neuroscience is something like an illformed art history sub-discipline that, rather than studying paintings from this or that historical period, studies the bottom third of paintings from all periods" (Grush 2003, p. 64). But this is not an apt analogy. Even the strongly interdisciplinary agenda I defend in this chapter can allow that in many contexts many of the brain's operations can still be relatively shielded from contextual influence: the extent of context-dependence, I argue, itself varies dramatically across contexts. The kinds of coordination, mediation, and reformatting of representations in 
which brains are involved, for example, offer more interesting patterns to study than would the geometrically defined subject matter of Grush's "ill-formed art history." Indeed, second-wave complementarity-based EM should specifically encourage attention to the unique roles played by brains in heterogeneous larger systems. See also Clark 2008, chapter 7, and my brief remarks in section 6 of this chapter on explanation in EM.

5. Although a detailed defense of this claim will have to await another occasion, I'd suggest that the interpretations of work in the cognitive psychology of memory, for example, offered by recent critics of EM (Rupert 2004; Adams and Aizawa 2008, pp. 63-68, pp. 137-139) do not fully and accurately characterize the whole diverse field. Not all research in the field treats intracranial processes as the only cognitive explananda; and not all research which does focus on intracranial processes does so to the exclusion of transcranial cognitive processes. For initial discussions of these issues in the case of memory see Wilson 2005; Tollefsen 2006; Barnier et al. 2008; Sutton 2009.

6. Adams and Aizawa (2008, chaps. 7-8) have, however, now discussed the complementarity argument: quoting my statement above of the complementarity principle, they note that they "agree with this completely," but deny that it supports EM (2008, p. 145). Since their discussion relies on an earlier draft of this current chapter, which has also been put to work in that earlier form by others, including defenders of EM like Menary (2006, 2007), Rowlands (2009), and Wilson (2005), I've thought it best to leave this first attempt at a systematic statement and defense of complementarity intact, postponing a full reply to Adams and Aizawa's deflationary reading to another occasion.

7. As this and other criticisms leveled at EM-such as those based on intrinsic content-reveal, the parity principle can also unhelpfully be read as leaving the inner as the standard of the cognitive, treating engrams in an individual brain as the unmarked case of the mental against which exograms must be judged. It's for this reason that Richard Menary rejects parity, because it seems to treat Otto's notebook as part of his cognitive system only due to its being coupled to him: "this is a residual form of internalism, because it assumes a discrete, already formed cognitive agent" (Menary 2006, p. 333). Clearly this isn't part of Clark's application of the parity principle: even so, it's notable that, responding to the kind of criticisms discussed in the text, Clark rightly resorts to considering "Otto-and-the-notebook" as "a single, integrated system" (2005a, p. 7). This, in my terms, is to shift from parity toward complementarity, from first- to second-wave EM.

8. Compare Bruno Latour's similar disclaimer: "in abandoning dualism our intent is not to throw everything into the same pot, to efface the distinct features of the various parts within the collective. We want analytical clarity, too, but following different lines than the one drawn for the polemical tug of war between subjects and objects" (Latour 1999, pp. 193-194). 
9. Richard Menary $(2006,2007$, this volume) calls this take on EM "cognitive integration," characterizing it as "the view that internal vehicles and processes are integrated with external vehicles and processes."

10. Here I'm drawing on, but also significantly revising, my own previous account of the memory arts (Sutton 2000). That paper has much more detail on this interpretation, with full reference to primary and secondary sources. My reading of the historical material there was particularly influenced by Stephen Greenblatt (1980) and Elaine Scarry (1988). The summary here for my current purposes is at a very general level, ideally to be filled out with studies of more specific contexts. In Sutton 2007 I have aligned this revised treatment of the arts of memory with a broader picture of the array of characteristic memory artifacts available in early modern Europe.

11. Gail Kern Paster characterizes humoralism as "a way of thinking about bodily behavior that . . . finds it much easier to account for a subject's moment-to-moment fluctuations in mood and action than to account for emotional steadiness and a high degree of psychological self-sameness. . . . Psychological self-sameness presupposes disembodied consciousness, not the humoral subject's full immersion in and continuous interaction with a constantly changing natural and cultural environment" (Paster 2004, p. 60). I survey psychological aspects of the holistic physiology of humors and fleeting animal spirits in Sutton 1998, chapter 2.

12. The story of the gradual rejection of animal spirits and of the reconstructive nature of memory in later seventeenth- and eighteenth-century philosophy and moral physiology is told in chapters 4-10 of my Philosophy and Memory Traces (Sutton 1998). A fuller prehistory of (and for) EM would incorporate that story into a larger narrative of the modern "invention of autonomy" (Schneewind 1997, pp. 3-11) and the correlative purifying "depsychologizing" of artifacts (Latour 1993; Jones and Stallybrass 2000; Sutton 2007).

13. In a footnote added to the second (2008) edition of her classic The Book of Memory, Carruthers acknowledges the relevance of Clark's work, suggesting that some of the medieval ideas she discusses are "in keeping with the extended-mind hypothesis of mental 'scaffolding,' exploited for the craft of thinking" (2008, p. 380). This is a neat demonstration of the two-way benefits which can flow from historical cognitive science, especially of the EM variety.

14. The second half of this quotation is highlighted in Bruno Latour's important celebratory review of Hutchins's "theory of computation by propagation of representational state" (Hutchins 1995, p. 230): Latour comments that "this means that there is nothing below the skin except the continuation of the same processes that go on outside" (Latour 1996, p. 58).

15. These points were powerfully made in some pre-EM philosophical engagements with the new dynamical approaches to cognition, for example by Jeff Foss 
(1992) and James Garson (1996). Dan Sperber's "epidemiology of representations" also embraces "a rather heterogeneous ontology, in that psychological and ecological phenomena are mixed together" (Sperber 1996, p. 26).

16. But note an interesting extremism in Adams and Aizawa's more recent treatment of this point. They question not only the "broad range of phenomena" which EM would address by studying (for example) "humans and computers, humans and books, humans and personal digital assistants, and who knows what else," but also the unity of a class which includes "human memory, canine memory, [and] mollusk memory" (Adams and Aizawa 2008, p. 141). This complaint is in tension with their wish elsewhere to rely firmly on scientific practice in the cognitive and neurosciences of memory, where carefully applied animal studies are entirely mainstream, providing vital evidence for key points of scientific consensus.

\section{References}

Adams, F., and Aizawa, K. (2001). The bounds of cognition. Philosophical Psychology, $14,43-64$.

Adams, F., and Aizawa, K. (2008). The Bounds of Cognition. Oxford: Blackwell.

Barnier, A. J., Sutton, J., Harris, C. B., and Wilson, R. A. (2008). A conceptual and empirical framework for the social distribution of cognition: The case of memory. Cognitive Systems Research, 9, 33-51.

Beckerman, B. (1962). Shakespeare at the Globe. New York: Macmillan.

Beer, R. (2000). Dynamical approaches to cognitive science. Trends in Cognitive Sciences, 4, 91-99.

Bernecker, S. (2010). Memory: A Philosophical Study. Oxford: Oxford University Press.

Butler, K. (1998). Internal Affairs: Making Room for Psychosemantic Internalism. Dordrecht: Kluwer.

Carruthers, M. (1990). The Book of Memory. Cambridge: Cambridge University Press (2nd edition, 2008).

Carruthers, M. (1998). The Craft of Thought. Cambridge: Cambridge University Press.

Carruthers, M., and Ziolkowski, J. (eds.) (2002). General introduction. In The Medieval Craft of Memory: An Anthology of Texts and Pictures (pp. 1-31). Philadelphia: Pennsylvania University Press.

Chalmers, D. (2008). Foreword to Clark, Supersizing the Mind. Oxford: Oxford University Press. 
Churchland, P. M. (1996). Flanagan on moral knowledge. In R. McCauley (ed.), The Churchlands and Their Critics. Oxford: Blackwell.

Clark, A. (1989). Microcognition: Philosophy, Cognitive Science, and Parallel Distributed Processing. Cambridge, MA: MIT Press.

Clark, A. (1993). Associative Engines: Connectionism, Concepts, and Representational Change. Cambridge, MA: MIT Press.

Clark, A. (1997). Being There: Putting Brain, Body, and World Together Again. Cambridge, MA: MIT Press.

Clark, A. (1998). Author's response: Review symposium on Being There. Metascience, 7, 95-103.

Clark, A. (2001a). Mindware: An Introduction to the Philosophy of Cognitive Science. Oxford: Oxford University Press.

Clark, A. (2001b). Reasons, robots, and the extended mind. Mind and Language, 16, 121-145.

Clark, A. (2002a). Towards a science of the bio-technological mind. International Journal of Cognitive Technology, 1, 21-33.

Clark, A. (2002b). The roots of "norm-hungriness." In H. Clapin (ed.), Philosophy of Mental Representation (pp. 37-43). Oxford: Oxford University Press.

Clark, A. (2003a). Natural-Born Cyborgs: Minds, Technologies, and the Future of Human Intelligence. Oxford: Oxford University Press.

Clark, A. (2003b). Forces, fields, and the role of knowledge in action: Commentary on Randall Beer. Adaptive Behavior, 11, 270-272.

Clark, A. (2004a). Is language special? Some remarks on control, coding, and co-ordination. Language Sciences, 26, 717-726.

Clark, A. (2004b). Author's response: Review symposium on Natural-Born Cyborgs. Metascience, 13, 169-181.

Clark, A. (2005a). Intrinsic content, active memory, and the extended mind. Analysis, 65, 1-11.

Clark, A. (2005b). Beyond the flesh: Some lessons from a mole cricket. Artificial Life, 11.

Clark, A. (2005c). Word, niche, and super-niche: How language makes minds matter more. Theoria, 20, 255-268.

Clark, A. (2007). Reinventing ourselves: The plasticity of embodiment, sensing, and mind. Journal of Medicine and Philosophy, 32, 263-282. 
Clark, A. (2008). Supersizing the Mind: Embodiment, Action, and Cognitive Extension. Oxford: Oxford University Press.

Clark, A., and Chalmers, D. (1998). The extended mind. Analysis, 58, 7-19. Reprinted as chapter 2 of this volume.

Coleman, J. (1992). Ancient and Medieval Memories. Cambridge: Cambridge University Press.

Dartnall, T. (2004). "We have always been . . cyborgs," review symposium on Clark, Natural-Born Cyborgs. Metascience, 13, 139-148.

Dartnall, T. (2005). Does the world leak into the mind? Active externalism, internalism, and epistemology. Cognitive Science, 29, 135-143.

Dennett, D. C. (2000). Making tools for thinking. In D. Sperber (ed.), Metarepresentations: A Multidisciplinary Perspective (pp. 17-29). Oxford: Oxford University Press.

Donald, M. (1991). Origins of the Modern Mind. Cambridge, MA: Harvard University Press.

Donald, M. (2001). A Mind So Rare: The Evolution of Human Consciousness. New York: W. W. Norton.

Foss, J. (1992). Introduction to the epistemology of the brain: Indeterminacy, micro-specificity, chaos, and openness. Topoi, 11, 45-57.

Garson, J. (1996). Cognition poised at the edge of chaos: A complex alternative to a symbolic mind. Philosophical Psychology, 9, 301-322.

Greenblatt, S. (1980). Renaissance Self-Fashioning. Chicago: Chicago University Press.

Grush, R. (2003). In defense of some "Cartesian" assumptions concerning the brain and its operation. Biology and Philosophy, 18, 53-93.

Haugeland, J. (1998). Mind embodied and embedded. In his Having Thought: Essays in the Metaphysics of Mind (pp. 207-237). Cambridge, MA: Harvard University Press.

Haugeland, J. (2002). Andy Clark on cognition and representation. In H. Clapin (ed.), Philosophy of Mental Representation (pp. 24-36). Oxford: Oxford University Press.

Hurley, S. (1998). Consciousness in Action. Cambridge, MA: Harvard University Press. Hutchins, E. (1995). Cognition in the Wild. Cambridge, MA: MIT Press.

Johnston, J. (2002). A future for autonomous agents: Machinic Merkwelten and artificial evolution. Configurations, 10, 473-516.

Jones, A. R., and Stallybrass, P. (2000). Renaissance Clothing and the Materials of Memory. Cambridge: Cambridge University Press. 
Kirsh, D. (2006). Distributed cognition: A methodological note. Pragmatics and Cognition, 14, 249-262.

Knappett, C. (2005). Thinking through Material Culture: An Interdisciplinary Approach. Philadelphia: University of Pennsylvania Press.

Knappett, C., and Malafouris, L. (eds.) (2008). Material Agency: Towards a Nonanthropocentric Approach. Berlin: Springer.

Kwint, M. (1999). Introduction: The physical past. In M. Kwint, C. Breward, and J. Aynsley (eds.), Material Memories (pp. 1-16). Oxford: Berg.

Latour, B. (1993). We Have Never Been Modern. Cambridge, MA: Harvard University Press.

Latour, B. (1996). Cogito ergo sumus! Or, Psychology swept inside out by the fresh air of the upper deck: Review of Hutchins 1995. Mind, Culture, and Activity, 3, 54-63.

Latour, B. (1999). A collective of humans and nonhumans. In Pandora's Hope: Essays on the Reality of Science Studies (pp. 174-215). Cambridge, MA: Harvard University Press..

Mackenzie, A. (2004). "We have always been . . cyborgs," review symposium on Clark, Natural-Born Cyborgs. Metascience, 13, 153-163.

McClelland, J. L., and Rumelhart, D. E. (1986). A distributed model of human learning and memory. In J. L. McClelland and D. E. Rumelhart (eds.), Parallel Distributed Processing: Explorations in the Microstructure of Cognition, volume 2 (pp. 170-215). Cambridge, MA: MIT Press.

Menary, R. (2006). Attacking the bounds of cognition. Philosophical Psychology, 19, 329-344.

Menary, R. (2007). Cognitive Integration: Mind and Cognition Unbounded. Basingstoke: Palgrave Macmillan.

Millikan, R. (2001). The language-thought partnership: A bird's eye view. Language and Communication, 21, 157-166.

Moè, A., and de Beni, R. (2005). Stressing the efficacy of the loci method: Oral presentation and the subject-generation of the loci pathway with expository passages. Applied Cognitive Psychology, 19, 95-106.

Noice, T., and Noice, H. (1997). The Nature of Expertise in Professional Acting: A Cognitive View. Mahwah, NJ: Lawrence Erlbaum.

O’Brien, G. (1998). Review symposium: Being There. Metascience, 7, 78-83.

Paster, G. K. (2004). Humoring the Body: Emotions and the Shakespearean Stage. Chicago: Chicago University Press. 
Poirier, P., and Chicoisne, G. (2006). A framework for thinking about distributed cognition. Pragmatics and Cognition, 14, 215-234.

Renfrew, C., Frith, C., and Malafouris, L. (eds.) (2009). The Sapient Mind: Archaeology meets Neuroscience. Oxford: Oxford University Press.

Renfrew, C., and Scarre, C. (eds.) (1999). Cognition and Material Culture: The Archaeology of Symbolic Storage. Cambridge: MacDonald Institute for Archaeological Research.

Richardson, A. (2004). Studies in literature and cognition: A field map. In A. Richardson and E. Spolsky (eds.), Cognition, Culture, and Complexity (pp. 1-30). Aldershot: Ashgate.

Ricoeur, P. (2004). Memory, History, Forgetting. Chicago: Chicago University Press.

Rogers, Y. (2006). Distributed cognition and communication. In K. Brown (ed.), Encyclopedia of Language and Linguistics, 2nd ed. (pp. 731-733). Amsterdam: Elsevier.

Rogers, Y., Scaife, M., and Rizzo, A. (2005). Interdisciplinarity: An emergent or engineered process? In S. J. Derry, M. A. Gernsbacher, and C. D. Schunn (eds.), Toward A Cognitive Science of Interdisciplinary Collaboration. Mahwah, NJ: Lawrence Erlbaum.

Rowlands, M. (1999). The Body in Mind: Understanding Cognitive Processes. Cambridge: Cambridge University Press.

Rowlands, M. (2003). Externalism: Putting Mind and World Back Together Again. Chesham: Acumen.

Rowlands, M. (2009). Extended cognition and the mark of the cognitive. Philosophical Psychology, 22, 1-19.

Rupert, R. (2004). Challenges to the hypothesis of extended cognition. Journal of Philosophy, 101, 389-428.

Scarry, E. (1988). Donne: "But yet the body is his booke." In E. Scarry (ed.), Literature and the Body (pp. 70-105). Baltimore: Johns Hopkins University Press.

Schneewind, J. B. (1997). The Invention of Autonomy. Cambridge: Cambridge University Press.

Sperber, D. (1996). Explaining Culture: A Naturalistic Approach. Oxford: Blackwell.

Stephenson, N. (1995). The Diamond Age. New York: Bantam Spectra.

Suchman, L. (1998). Human/machine reconsidered. Cognitive Studies, 5, 5-13.

Sutton, J. (1998). Philosophy and Memory Traces: Descartes to Connectionism. Cambridge: Cambridge University Press.

Sutton, J. (2000). Body, mind, and order: Local memory and the control of mental representations in medieval and Renaissance sciences of self. In G. Freeland and 
A. Corones (eds.), 1543 and All That: Word and Image in the Proto-Scientific Revolution (pp. 117-150). Dordrecht: Kluwer.

Sutton, J. (2002a). Porous memory and the cognitive life of things. In D. Tofts, A. Jonson, and A. Cavallaro (eds.), Prefiguring Cyberculture: An Intellectual History (pp. 130-141). Cambridge, MA, and Sydney: MIT Press and Power Publications.

Sutton, J. (2002b). Cognitive conceptions of language and the development of autobiographical memory. Language and Communication, 22, 375-390.

Sutton, J. (2004). Representation, reduction, and interdisciplinarity in the sciences of memory. In H. Clapin, P. Staines, and P. Slezak (eds.), Representation in Mind: New Approaches to Mental Representation (pp. 187-216). Amsterdam: Elsevier.

Sutton, J. (2006). Distributed cognition: Domains and dimensions. Pragmatics and Cognition, 14, 235-247.

Sutton, J. (2007). Spongy brains and material nemories. In M. Floyd-Wilson and G. Sullivan (eds.), Embodiment and Environment in Early Modern Europe (pp. 14-34). London: Palgrave.

Sutton, J. (2008). Material agency, skills, and history: Distributed cognition and the archaeology of memory. In L. Malafouris and C. Knappett (eds.), Material Agency: Towards a Non-anthropocentric Approach (pp. 37-55). Berlin: Springer.

Sutton, J. (2009). Remembering. In P. Robbins and M. Aydede (eds.), The Cambridge Handbook of Situated Cognition (pp. 217-235). Cambridge: Cambridge University Press.

Tollefsen, D. P. (2006). From extended mind to collective mind. Cognitive Systems Research, 7, 140-150.

Tooby, J., and Cosmides, L. (1992). The psychological foundations of culture. In J. H. Barkow, L. Cosmides, and J. Tooby (eds.), The Adapted Mind (pp. 19-136). Oxford: Oxford University Press.

Tribble, E. (2005). Distributing cognition in the globe. Shakespeare Quarterly, 56, 135-155.

Tribble, E. (2006). "The dark backward and abysm of time": The Tempest and memory. College Literature, 33, 151-168.

von Eckardt, B. (2001). Multidisciplinarity and cognitive science. Cognitive Science, 21, 453-470.

Wegner, D. M. (1987). Transactive memory: A contemporary analysis of group mind. In B. Mullen and G. R. Goethals (eds.), Theories of Group Behavior (pp. 185208). New York: Springer-Verlag.

Wegner, D. M., Erber, R., and Raymond, P. (1991). Transactive memory in close relationships. Journal of Personality and Social Psychology, 61, 923-929. 
Weiskopf, Daniel A. (2008). Patrolling the mind's boundaries. Erkenntnis, 68(2), 265-276.

Wheeler, M. (2004). Is language the ultimate artefact? Language Sciences, 26, 693-715.

Wheeler, M., and Clark, A. (2008). Culture, embodiment, and genes: Unravelling the triple helix. Philosophical Transactions of the Royal Society Series B, 363, 3563-3575.

Wilson, R. A. (2004). Boundaries of the Mind: The Individual in the Fragile SciencesCognition. Cambridge: Cambridge University Press.

Wilson, R. A. (2005). Collective memory, group minds, and the extended mind thesis. Cognitive Processing, 6, 227-236.

Wilson, R. A., and Clark, A. (2009). How to situate cognition: Letting nature take its course. In P. Robbins and M. Aydede (eds.), The Cambridge Handbook of Situated Cognition. Cambridge: Cambridge University Press.

Yates, F. (1966). The Art of Memory. London: Routledge and Kegan Paul. 